九州大学学術情報リポジトリ

Kyushu University Institutional Repository

\title{
AN INFORMATION THEORY OF GAME SYSTEMS
}

Kai, Yu

Research Institute of Fundamental Information Science, Kyushu University

Kano, Seigo

Research Institute of Fundamental Information Science, Kyushu University

https://doi.org/10.5109/13150

出版情報: 統計数理研究. 19 (3/4)，pp.87-102，1981-03. Research Association of Statistical Sciences

バージョン：

権利関係 : 


\title{
AN INFORMATION THEORY OF GAME SYSTEMS
}

\author{
By
}

\author{
Y û KAI* and Seigo KAN $\overline{0}^{* *}$
}

(Received November 15, 1980)

\begin{abstract}
We define non-cooperative game systems and cooperative game systems from a viewpoint of information theory. Concerning the both systems we show some sufficient conditions under which players can obtain the total information on the other players' strategies from a sequence of observations. In a cooperative case we give a numerical model and calculate the amounts of information obtained from the sequential observations by the players in a cooperative group.
\end{abstract}

\section{Introduction.}

Generally in the game theory the objects of the studies are players' rewards or costs. For example in the stochastic games one of the most essential problems is to find the optimal strategies which give the equilibrium points concerning the players' rewards. However, considering usual games played by more than or equal to two players, like as chess, bridge, etc., we must not overlook the role of the information concerning the players' strategies. Practically in such a game, if a player obtains enough information to estimate the other players' strategies but does not give the others sufficient information on his strategies, the player can win the game easily. Therefore we try to treat the games from the viewpoint of the information theory. In the present paper we deal with the games played through infinitely many stages and study the amounts of information that the players obtain about the other players' strategies from sequential observations on the players' states.

At the beginning of a game every player chooses one of his strategies by some system independent of the other players. The strategies chosen by the players determine players' actions at every stage, and the actions determine the players' states. At each stage the states or both of the states and the actions are observed by every player. For the sake of brevity we call simply the observed phenomena the states. We suppose that every player's strategy is determined by a probability distribution and suppose further that the players' states are determined by conditional distributions according to their strategies. By the suppositions each player can obtain some infor-

*,** Research Institute of Fundamental Information Science, Kyushu University, Fukuoka 
mation about the other players' strategies from the observations on the sequence of the states. The problem in the present paper is how much amounts of information a player can obtain on the other palyers' strategies from the sequential observations. We study the problem by applying the results of Rényi [1], [2] and Korsh [3] concerning the testing hypothesis theory, which gave some conditions under which the total information on a parameter will be obtained from sequential observations.

In section 2 we consider two person games where both players do not cooperate with each other. We give some sufficient conditions under which a player can obtain the total information on the other player's strategy from the sequential observations. Furthermore we show some examples in the two cases that the sequential states of the players are indendent and normally distributed and that are Markov processes. In section 3 we study four person games where players are allowed to cooperate with each other. We define the cooperation as the behaviors that every player in the cooperative group gives his partners all the information that he has obtained. We give sufficient conditions under which a player in the cooperation can obtain the total information on the other players' strategies from the sequential observat ions on the players' states. We also give numerical models of the games and show some tables of the amounts of information obtained from the observations. In the last section we discuss the hierarchy of information games and consider this paper's results from the viewpoint of application to brain information processing considered as unified information processing, parallel processing or learning processes.

\section{Information in non-cooperative game systems.}

In this section we study the amounts of information that players in non-cooperative game systems obtain on the other players' strategies from sequential observations. For the sake of brevity we only deal with two person game systems, which are defined as follows. Let $A=(\Omega, \mathcal{A}, P)$ be a probability space, i. e. $\Omega$ an arbitrary nonempty set, $\AA$ an $\sigma$-algebra of subsets of $\Omega$ and $P$ a probability measure on $A$. Let $\theta_{i}(i=1,2)$ be a random parameter whose possible values denote player $i$ 's strategies. We suppose that for every player the set of his possible strategies is finite. We suppose further that $\theta_{1}$ and $\theta_{2}$ are independent, and denote their values by $\left\{\alpha_{1}, \cdots, \alpha_{n}\right\}$ and $\left\{\beta_{1}, \cdots, \beta_{m}\right\}$ respectively. We use the following notations:

$$
p_{k}=P\left(\left\{\omega \in \Omega: \theta_{1}(\omega)=\alpha_{k}\right\}\right)
$$

and

$$
q_{l}=P\left(\left\{\omega \in \Omega: \theta_{2}(\omega)=\beta_{l}\right\}\right) .
$$

The entropy of $\theta_{1}$ is defined by Shannon's formula

$$
H\left(\theta_{1}\right)=\sum_{k=1}^{n} p_{k} \log \frac{1}{p_{k}}
$$

and $H\left(\theta_{2}\right)$ is defined similarly.

Let $\left\{\left(X_{t}, Y_{t}\right)\right\}=\left\{\left(X_{t}(\omega), Y_{t}(\omega)\right)\right\},(t=1,2, \cdots)$ be a sequence of 2-dimensional random vectors, and let $X_{t}$ and $Y_{t}$ denote player 1's state and player 2's state at the $t$-th stage respectively, which can be observed by both players. We introduce the notation 
$\xi_{t}=\xi_{t}(\omega)=\left(\left(X_{1}(\omega), Y_{1}(\omega)\right), \cdots,\left(X_{t}(\omega), Y_{i}(\omega)\right)=R^{2 t}\right.$ and suppose that the probability distribution of $\xi_{t}$ is absolutely continuous for all $t=1,2, \cdots$, so we denote by $\dot{Q}_{\mathrm{k}}\left(\xi_{t}\right)$ the density function of $\xi_{t}$ under the condition that $\theta_{1}=\alpha_{k}$ and $\theta_{2}=\beta_{l}$. We denote further by $\dot{\psi}_{l}^{(t)}\left(\xi_{t}\right)$ the density function of $\xi_{t}$ under the condition $\theta_{2}=\beta_{l}$ and denote by $\Phi^{(t)}\left(\xi_{t}\right)$ the density function of $\hat{s}_{t}$, then we have easily

$$
\varphi_{l}^{(t)}\left(\xi_{t}\right)=\sum_{k} p_{k} \phi_{k l}^{(t)}\left(\hat{\xi}_{t}\right)
$$

and

$$
\Phi^{(t)}\left(\xi_{t}\right)=\sum_{i} \sum_{l} p_{k} q_{l} \phi_{k l}^{(t)}\left(\xi_{t}\right)
$$

Every player observes $\xi_{t}$ and want to know the other player's strategy, i. e. the parameter's value. Thus we study under what conditions on $\left\{\phi_{k l}^{(t)}\right\}$ a player can obtain the total information about the other player's strategy from $\xi_{t}$. To avoid complexity we treat only the information that player 1 can obtain about player 2's strategy. Concerning the information that player 2 can obtain on player 1's strategy, we can show the same results as those in this section.

Let $I_{1: 2}(t)$ denote the amount of information that player 1 obtains concerning $\theta_{2}$ from $\xi_{t}$. Then we have

$$
I_{1: 2}(t)=H\left(\theta_{2}\right)-E\left[H\left(\theta_{2} \mid \xi_{t}\right)\right]
$$

where $H\left(\theta_{2} \mid \xi_{t}\right)$ is the conditional entropy of $\theta_{2}$ when $\xi_{t}$ is given, i. e.

$$
H\left(\theta_{2} \mid \xi_{t}\right)=\sum_{l} P\left(\theta_{2}=\beta_{l} \mid \xi_{t}\right) \log \frac{1}{P\left(\theta_{2}=\beta_{l} \mid \xi_{t}\right)},
$$

and $E\left[H\left(\theta_{2} \mid \xi_{t}\right)\right]$ means the expectation of $H\left(\theta_{2} \mid \xi_{t}\right)$, i. e.

$$
E\left[H\left(\theta_{2} \mid \xi_{t}\right)\right]=\int_{R^{2 t}} H\left(\theta_{2} \mid \xi_{t}\right) \Phi^{(t)}\left(\xi_{t}\right) d \xi_{t} .
$$

Throughout this paper we denote by log logarithm with base 2 . By the suppositions and the Bayes' theorem we have easily

$$
\begin{aligned}
P\left(\theta_{2}=\beta_{l} \mid \xi_{t}\right) & =\frac{q_{l} \phi_{l}^{(t)}\left(\xi_{t}\right)}{\Phi^{(t)}\left(\xi_{t}\right)} \\
& =\frac{\sum_{k} p_{k} q_{l} \phi_{k l}^{(t)}\left(\xi_{t}\right)}{\Phi^{(t)}\left(\xi_{t}\right)} .
\end{aligned}
$$

It is easy to see that $I_{1: 2}(t)$ is nondecreasieg for $t=1,2, \cdots$ and $I_{1: 2}(t) \leqq H\left(\theta_{2}\right)$. Thus, if $\lim _{t \rightarrow \infty} I_{1: 2}(t)=H\left(\theta_{2}\right)$, we say that player 1 obtains the total information on $\theta_{2}$ from the sequential observations $\left\{\xi_{t}\right\}$.

We know an essential lemma of Rényi [1].

LEMMA 1. There exists a universal constant $C>0$ such that for any sequence $a_{1}, a_{2}, \cdots, a_{N}$ of positive numbers forming a probability distribution (i.e. $a_{1}+\cdots a_{N}=1$ ) we have 


$$
\sum_{i=1}^{N} a_{i} \log \frac{1}{a_{i}} \leqq C \sum_{i=2}^{N} \sqrt{a_{i}}
$$

Applying this lemma, we prove the following result.

THEOREM 1. There exists a universal constant $C>0$ such that for all $t(t=1,2, \cdots)$

$$
\begin{aligned}
& E\left[H\left(\theta_{2} \mid \xi_{t}\right)\right] \\
\leqq & C \sum_{k} \sum_{j} \sum_{l} \sum_{h \neq l} \int_{R^{2 t}}\left\{\phi_{k l}^{(t)}\left(\xi_{t}\right) \phi_{j h}^{(t)}\left(\xi_{t}\right)\right\}^{1 / 2} d \xi_{t} .
\end{aligned}
$$

Proof. By (2) and Lemma 1 there exists a constant $C>0$ such that for all $l$, $(l=1, \cdots, m)$ and all $t,(t=1,2, \cdots)$

$$
H\left(\theta_{2} \mid \xi_{t}\right) \leqq C \sum_{h \neq l}\left(P\left(\theta_{2}=\beta_{h} \mid \xi_{t}\right)\right)^{1 / 2} .
$$

Then from (3) and (4) we have for all $t,(t=1,2, \cdots)$

$$
\begin{aligned}
& E\left[H\left(\theta_{2} \mid \xi_{t}\right)\right] \\
\leqq & C \int_{R^{2} t}\left\{\sum_{h \neq l}\left(P\left(\theta_{2}=\beta_{h} \mid \xi_{t}\right)\right)^{1 / 2}\right\} \Phi^{(t)}\left(\xi_{t}\right) d \xi_{t} \\
= & C \int_{R^{2 t}} \sum_{h \neq l}\left\{\frac{\sum_{j} p_{j} q_{h} \phi_{j h}^{(t)}\left(\xi_{t}\right)}{\Phi^{(t)}\left(\xi_{t}\right)}\right\}^{1 / 2} \Phi^{(t)}\left(\xi_{t}\right) d \xi_{t} \\
= & C \int_{R^{2 t}} \sum_{h \neq l}\left\{\sum_{j} p_{j} q_{h} \phi_{j h}^{(t)}\left(\xi_{t}\right)\right\}^{1 / 2}\left\{\sum_{k} \sum_{l} p_{k} q_{l} \phi_{k l}^{(t)}\left(\xi_{t}\right)\right\}^{1 / 2} d \xi_{t} \\
\leqq & C \sum_{k} \sum_{j} \sum_{l} \sum_{h \neq l}\left(p_{k} p_{j} q_{l} q_{h}\right)^{1 / 2} \int_{R^{2 t}}\left\{\phi_{k l}^{(t)}\left(\xi_{t}\right) \phi_{j h}^{(t)}\left(\xi_{t}\right)\right\}^{1 / 2} d \xi_{t} \\
\leqq & C \sum_{k} \sum_{j} \sum_{l} \sum_{h \neq l} \int_{R^{2 t}}\left\{\phi_{k l}^{(t)}\left(\xi_{t}\right) \phi_{j h}^{(t)}\left(\xi_{t}\right)\right\}^{1 / 2} d \xi_{t} .
\end{aligned}
$$

In the following two cases we consider the sufficient conditions under which player 1 can obtain the total information on $\theta_{2}$ from $\left\{\xi_{t}\right\}$.

\section{A. Amount of information in independent case.}

We suppose that under conditions $\theta_{1}=\alpha_{k}$ and $\theta_{2}=\beta_{l}$ the random vectors $\left(X_{1}, Y_{1}\right)$, $\left(X_{2}, Y_{2}\right), \cdots$ are independent. For each $t,(t=1,2, \cdots)$ we denote by $f_{k l}^{(t)}(x, y)$ the density function of the random vector $\left(X_{t}, Y_{t}\right)$ under the conditions that $\theta_{1}=\alpha_{k}$ and $\theta_{2}=\beta_{l}$. Then the following result is easily shown by Theorem 1 .

THEOREM 2. If for every $k, j, l$ and $h \neq l$

$$
\lim _{t \rightarrow \infty} \prod_{\nu=1}^{t} \int_{R^{2}}\left\{f_{k l}^{(\nu)}(x, y) f_{j h}^{(\nu)}(x, y)\right\}^{1 / 2} d x d y=0,
$$

then player 1 can obtain the total information on $\theta_{2}$ from $\left\{\xi_{t}\right\}$, that is

$$
\lim _{t \rightarrow \infty} I_{1: 2}(t)=H\left(\theta_{2}\right) \text {. }
$$


Proof. As $\left\{\left(X_{t}, Y_{t}\right)\right\},(t=1,2, \cdots)$ are independent, for each $k$ and $l$ we have

$$
\phi_{k l}^{(t)}\left(\xi_{l}\right)=\prod_{\nu=1}^{t} f_{k l}^{(\mu)}\left(X_{\nu}, Y_{\nu}\right) \text {. }
$$

Then by Theorem 1 we have

$$
\begin{aligned}
& E\left[H\left(\theta_{2} \mid \xi_{t}\right)\right] \\
\leqq & C \sum_{k} \sum_{j} \sum_{l} \sum_{h \neq l} \int_{R^{2 t}}\left\{\prod_{\nu=1}^{t} f_{k l}^{(L)}\left(x_{\nu}, y_{\nu}\right) \prod_{\nu=1}^{t} f_{j h}^{(\nu)}\left(x_{\nu}, y_{\nu}\right)\right\}^{1 / 2} d x_{1} d y_{1} \cdots d x_{t} d y_{t} \\
= & C \sum_{k} \sum_{j} \sum_{l} \sum_{h \neq l} \prod_{\nu=1}^{t} \int_{R^{2 t}}\left\{f_{k l}^{(\nu)}(x, y) f_{j h}^{(\nu)}(x, y)\right\}^{1 / 2} d x d y .
\end{aligned}
$$

Thus under the condition of this theorem we have

$$
\lim _{t \rightarrow \infty} I_{1: 2}(t)=H\left(\theta_{2}\right) .
$$

EXAmple 1. Let $\left(X_{t}, Y_{t}\right),(t=1,2, \cdots)$, under conditions $\theta_{1}=\alpha_{k}$ and $\theta_{2}=\beta_{l}$, have 2-dimensional normal distribution such that

$$
\begin{aligned}
f_{k l}^{(t)}(x, y)= & \frac{1}{2 \pi \sigma_{t} \delta_{t} \sqrt{ } 1-\rho_{t}^{2}} \exp \left[-\frac{1}{2\left(1-\rho_{t}^{2}\right)}\left\{\frac{(x-u(k, l))^{2}}{\sigma_{t}^{2}}\right.\right. \\
& \left.\left.-2 \rho_{t} \frac{(x-u(k, l))(y-v(k, l))}{\sigma_{t} \delta_{t}}+\frac{(y-v(k, l))^{2}}{\delta_{t}^{2}}\right\}\right] .
\end{aligned}
$$

Then we have

$$
\begin{aligned}
& \int_{R^{2}}\left\{f_{k l}^{(t)}(x, y) f_{j h}^{(t)}(x, y)\right\}^{1 / 2} d x d y \\
= & \int_{R^{2}} \frac{1}{2 \pi \sigma_{t} \delta_{t} \sqrt{ } 1-\rho_{t}^{2}} \exp \left[-\frac{1}{2\left(1-\rho_{t}^{2}\right)}\left\{\frac{\left(x-\alpha_{k j l h}\right)^{2}}{\sigma_{t}^{2}}\right.\right. \\
& -\frac{\left[2 \rho_{t}\right.}{\sigma_{t} \delta_{t}}\left(x-\alpha_{k j l h}\right)\left(y-\beta_{k j l h}\right)+\frac{\left(y-\beta_{k j l h}\right)^{2}}{\delta_{t}^{2}} \\
& \left.\left.+\left(\frac{a_{k j l h}}{2 \sigma_{t}}\right)^{2}-\frac{\rho_{t}}{2 \sigma_{t} \delta_{t}} a_{k j l h} b_{k j l h}+\left(\frac{b_{k j l h}}{2 \delta_{t}}\right)^{2}\right\}\right] d x d y \\
= & \exp \left[-\frac{1}{8\left(1-\rho_{t}^{2}\right)}\left\{\left(\frac{a_{k j l h}}{\sigma_{t}}\right)^{2}-\frac{2 \rho_{t}}{\sigma_{t} \delta_{t}} a_{k j l h} b_{k j l h}+\left(\frac{b_{k j l h}}{\delta_{t}}\right)^{2}\right\}\right],
\end{aligned}
$$

where

$$
\begin{aligned}
& \alpha_{k j l h}=\frac{u(k, l)+u(j, h)}{2}, \quad \beta_{k j l h}=\frac{v(k, l)+v(j, h)}{2}, \\
& a_{k j l h}=u(k, l)-u(j, h) \text { and } \quad b_{k j l h}=v(k, l)-v(j, h)
\end{aligned}
$$

(a) Case that $a_{k j l h} b_{k j l h} \geqq 0$.

Since $\left|\rho_{t}\right| \leqq 1$ for all $t=1,2, \cdots$, we have

$$
\left(\frac{a_{k j l h}}{\sigma_{t}}\right)^{2}-\frac{2 \rho_{t}}{\sigma_{t} \delta_{t}} a_{k j l h} b_{k j l h}+\left(\frac{b_{k j l h}}{\delta_{t}}\right)^{2} \geqq\left\{\frac{a_{k j l h}}{\sigma_{t}}-\frac{b_{k j l h}}{\delta_{t}}\right\}^{2} .
$$


Thus we have

$$
\begin{gathered}
\int_{R^{2}}\left\{f_{k l}^{(t)}(x, y) f_{j h}^{(t)}(x, y)\right\}^{1,2} d x d y \\
\leqq \\
\exp \left[-\frac{1}{8\left(1-\rho_{t}^{2}\right)}\left\{\frac{a_{k j l h}}{\sigma_{t}}-\frac{b_{k j l h}}{\delta_{t}}\right\}^{2}\right] .
\end{gathered}
$$

Hence, if for every $k, j, l$ and $h \neq l$

$$
\sum_{i=1}^{\infty} \frac{1}{1-\rho_{t}^{2}}\left\{\frac{a_{k j l h}}{\sigma_{t}}-\frac{b_{k j l h}}{\delta_{t}}\right\}^{2}=\infty,
$$

we have

$$
\lim _{t \rightarrow \infty} \prod_{\nu=1}^{t} \int_{R^{2}}\left\{f_{k l}^{(-)}(x, y) f_{j h}^{(L)}(x, y)\right\}^{1 / 2} d x d y=0 .
$$

Then by Theorem 2

$$
\lim _{t \rightarrow \infty} I_{1: 2}(t)=H\left(\theta_{2}\right) .
$$

(b) Case that $a_{k j l h} b_{k j l h}<0$.

We can show similarly that for all $t=1,2, \cdots$,

$$
\begin{gathered}
\int_{R^{2}}\left\{f_{k l}^{(t)}(x, y) f_{j h}^{(t)}(x, y)\right\}^{1 / 2} d x d y \\
\leqq \\
\exp \left[-\frac{1}{8\left(1-\rho_{t}^{2}\right)}\left\{\frac{a_{k j l h}}{\sigma_{t}}+\frac{b_{k j l h}}{\delta_{t}}\right\}^{2}\right] .
\end{gathered}
$$

Thus, if for every $k, j, l$ and $h \neq l$

$$
\sum_{t=1}^{\infty} \frac{1}{1-\rho_{t}^{2}}\left\{\frac{a_{k j l h}}{\sigma_{t}}-\frac{b_{k j l h}}{\delta_{t}}\right\}^{2}=\infty,
$$

we have

$$
\lim _{t \rightarrow \infty} \prod_{\nu=1}^{t} \int_{R^{2}}\left\{f_{k l}^{(L)}(x, y) f_{j h}^{(\nu)}(x, y)\right\}^{1 / 2} d x d y=0 .
$$

Then by Theorem 2

$$
\lim _{t \rightarrow \infty} I_{1: 2}(t)=H\left(\theta_{2}\right) .
$$

\section{B. Amount of information in Markovian case.}

We suppose that the random process $\left\{\left(X_{t}, Y_{t}\right)\right\},(t=1,2, \cdots)$, under conditions $\theta_{1}=\alpha_{k}$ and $\theta_{2}=\beta_{l}$, is a denumerable state Markov chain. We use the following notations:

and

$$
P_{k l}^{(0)}\left(X_{1}, Y_{1}\right)=P\left(X_{1}, Y_{1} \mid \theta_{1}=\alpha_{k}, \theta_{2}=\beta_{l}\right)
$$

$$
P_{k l}^{(t)}\left(X_{t+1}, Y_{t+1} \mid X_{t}, Y_{t}\right)=P\left(X_{t+1}, Y_{t+1} \mid \theta_{1}=\alpha_{k}, \theta_{2}=\beta_{l}, X_{t}, Y_{t}\right) .
$$

Then we have

$$
\begin{aligned}
& P\left(\xi_{t} \mid \theta_{1}=\alpha_{k}, \theta_{2}=\beta_{l}\right) \\
= & P_{k l}^{(@)}\left(X_{1}, Y_{1}\right) P_{k l}^{(1)}\left(X_{2}, Y_{2} \mid X_{1}, Y_{1}\right) \cdots P_{k l}^{(t-1)}\left(X_{t}, Y_{t} \mid X_{t-1}, Y_{t-1}\right) .
\end{aligned}
$$


We define the following quantity:

$$
\begin{aligned}
\delta_{k j l h}^{(t)}= & \sup _{\left\langle X_{t}, Y_{t}\right.}, X_{t+1, Y_{t+1}}\left\{P_{k i}^{(t)}\left(X_{t+1}, Y_{t+1} \mid X_{t}, Y_{t}\right)\right. \\
& \left.\cdot P_{j h}^{(t)}\left(X_{t+1}, Y_{t+1} \mid X_{t}, Y_{t}\right)\right\}^{\}^{1 / 2}} .
\end{aligned}
$$

Then the following result is proved.

THEOREM 3. If for every $k, j, l$ and $h \neq l$

$$
\prod_{t=1}^{\infty} \delta_{k j l i}^{(t)}=0
$$

player 1 can obtain the total information on $\theta_{2}$ from $\{\hat{s}\}$, that is

$$
\lim _{t \rightarrow \infty} I_{1: 2}(t)=H\left(\theta_{2}\right) .
$$

Proof. By Lemma 1 for any $l,(l=1,2, \cdots, m)$ there exists a constant $C>0$ such that

$$
H\left(\theta_{2} \mid \xi_{t}\right) \leqq C \sum_{n \neq l} \sqrt{P\left(\theta_{2}=\beta_{h} \mid \xi_{t}\right)}
$$

By the Bayes' theorem

$$
\begin{aligned}
P\left(\theta_{2}=\beta_{h} \mid \xi_{t}\right) & =\frac{q_{h} P\left(\xi_{t} \mid \theta_{2}=\beta_{h}\right)}{P\left(\xi_{t}\right)} \\
& =\frac{\sum_{j} p_{j} q_{h} P\left(\xi_{t} \mid \theta_{1}=\alpha_{j}, \theta_{2}=\beta_{h}\right)}{\sum_{k} \sum_{l} p_{k} q_{l} P\left(\xi_{t} \mid \theta_{1}=\alpha_{k}, \theta_{2}=\beta_{l}\right)} .
\end{aligned}
$$

Then

$$
\begin{aligned}
& E\left[H\left(\theta_{2} \mid \xi_{t}\right)\right] \\
= & \sum_{\xi_{t}} P\left(\xi_{t}\right) H\left(\theta_{2} \mid \xi_{t}\right) \\
\leqq & C \sum_{\xi t} P\left(\xi_{t}\right) \sum_{h \neq l}\left\{\frac{\sum_{j} p_{j} q_{h} P\left(\xi_{t} \mid \theta_{1}=\alpha_{j}, \theta_{2}=\beta_{h}\right)}{P\left(\xi_{t}\right)}\right\}^{1 / 2} \\
= & C \sum_{\xi t}\left\{\sum_{k} \sum_{l} p_{k} q_{l} P\left(\xi_{t} \mid \theta_{1}=\alpha_{k}, \theta_{2}=\beta_{l}\right)\right\}^{1 / 2} \sum_{h \neq l}\left\{\sum_{j} p_{j} q_{h} P\left(\xi_{t} \mid \theta_{1}=\alpha_{j}, \theta_{2}=\beta_{h}\right)\right\}^{1 / 2} \\
\leqq & C \sum_{\xi t}\left\{\sum_{k} \sum_{l}\left(p_{k} q_{l} P\left(\xi_{l} \mid \theta_{1}=\alpha_{k}, \theta_{2}=\beta_{l}\right)\right)^{1 / 2}\right\}\left\{\sum_{j} \sum_{h \neq l}\left(p_{j} q_{h} P\left(\xi_{t} \mid \theta_{1}=\alpha_{j}, \theta_{2}=\beta_{h}\right)\right)^{1 / 2}\right\} \\
\leqq & C \sum_{k} \sum_{j} \sum_{l} \sum_{h \neq l}\left(p_{k} q_{l} p_{j} q_{h}\right)^{1 / 2} \sum_{\xi_{t}}\left\{P\left(\xi_{t} \mid \theta_{1}=\alpha_{k}, \theta_{2}=\beta_{l}\right) P\left(\xi_{t} \mid \theta_{1}=\alpha_{j}, \theta_{2}=\beta_{h}\right)\right\}^{1 / 2} \\
\leqq & C \sum_{k} \sum_{j} \sum_{l} \sum_{h \neq l} \sum_{\xi_{l}}\left\{P_{k l}^{(0)}\left(X_{1}, Y_{1}\right) P_{j h}^{(0)}\left(X_{1}, Y_{1}\right)\right\}^{1 / 2} \\
& \cdot \prod_{\nu=1}^{t-1}\left\{P_{k l}^{(\nu)}\left(X_{\nu+1}, Y_{\nu+1} \mid X_{\nu}, Y_{\nu}\right) P_{j h}^{(\nu)}\left(X_{\nu+1}, Y_{\nu+1} \mid X_{\nu}, Y_{\nu}\right)\right\}^{1 / 2}
\end{aligned}
$$


$\leqq C \sum_{k} \sum_{j} \sum_{l} \sum_{h \neq l} \prod_{\nu=1}^{t-1} \delta_{k l j h}^{(L)}$

Thus, if for every $k, h, l$ and $h \neq l$

$$
\lim _{t \rightarrow \infty} \prod_{\nu=1}^{t} \delta_{k l j h}^{(2)}=0
$$

we have

$$
\begin{aligned}
\lim _{t \rightarrow \infty} I_{1: 2}(t) & =\lim _{t \rightarrow \infty}\left\{H\left(\theta_{2}\right)-E\left[H\left(\theta_{2} \mid \hat{\xi}_{t}\right)\right\}\right. \\
& =H\left(\theta_{2}\right) .
\end{aligned}
$$

ExAmple 2. Let $\left\{\left(X_{t}, Y_{t}\right)\right\},(t=1,2, \cdots)$ under conditions $\theta_{1}=\alpha_{k}$ and $\theta_{2}=\beta_{l}$ be a stationary Markov process with a finite state set $\left\{a_{1}, \cdots, a_{M}\right\}$ which is a subset of 2 -dimensional vector space. Since the process is stationary, we put for all $t=1,2, \cdots$,

$$
q_{k l}(r, s)=P_{k l}^{(t)}\left(\left(X_{t+1}, Y_{t+1}\right)=a_{s} \mid\left(X_{t}, Y_{t}\right)=a_{r}\right) .
$$

Thus we have by (5) for all $t=1,2, \cdots$,

$$
\delta_{k l j h}^{(t)}=\max _{r} \sum_{s}\left\{q_{k l}(r, s) q_{j h}(r, s)\right\}^{1 / 2} .
$$

As the process is stationary we put $\hat{o}_{k j l h}=\delta_{k j l h}^{(t)}$ for all $t=1,2, \ldots$. Then, if for every $k, j, l$ and $h \neq l$

$$
\left(q_{k l}(r, 1), \cdots, q_{k l}(r, M)\right) \neq\left(q_{j h}(r, 1), \cdots, q_{j h}(r, M)\right)
$$

for all $r=1,2, \cdots, M$, we see easily $\hat{o}_{k j l h}<1$. Therefore by Tneorem 3 we have

$$
\lim _{t \rightarrow \infty} I_{1: 2}(t)=H\left(\theta_{2}\right) .
$$

\section{Information in cooperative game systems.}

In this section we consider cooperation in game systems from the viewpoint of information theory. Players in the game systems are allowed to cooperate with each other. Then we study the amounts of information that the players in a cooperative group can obtain on the strategies of the players, who are not included in the group, from sequential observations. For the sake of brevity we deal with four person game systems, which are defined as follows. Let $A=(\Omega, \mathcal{A}, P)$ be a probability space and $\theta_{i}=\theta_{i}(\omega),(\omega \in \Omega$ and $i=1,2,3,4)$, be a random parameter whose possible values denote player $i$ 's strategies. We suppose that the set of each player's possible strategies is finite and suppose further that $\theta_{1}, \theta_{2}, \theta_{3}$ and $\theta_{4}$ are independent each other. We denote the values of $\theta_{1}, \theta_{2}, \theta_{3}$ and $\theta_{4}$ by $\left\{\alpha_{1}, \cdots, \alpha_{N_{1}}\right\},\left\{\beta_{1}, \cdots, \beta_{N_{2}}\right\},\left\{\gamma_{1}, \cdots, \gamma_{N_{3}}\right\}$ and $\left\{\delta_{1}, \cdots, \delta_{N_{4}}\right\}$ respectively. We put for each $i, j, k$ and $l$

$$
\begin{aligned}
p_{i j k l}= & P\left(\left\{\omega \in \Omega: \theta_{1}(\omega)=\alpha_{i}\right\}\right) P\left(\left\{\omega \in \Omega: \theta_{2}(\omega)=\beta_{j}\right\}\right) \\
& \cdot P\left(\left\{\omega \in \Omega: \theta_{3}(\omega)=\gamma_{k}\right\}\right) P\left(\left\{\omega \in \Omega: \theta_{4}(\omega)=\delta_{l}\right\}\right) .
\end{aligned}
$$

Let $\left\{\left(X_{t}, Y_{t}, Z_{t}, W_{t}\right)\right\}=\left\{\left(X_{t}(\omega), Y_{t}(\omega), Z_{t}(\omega), W_{t}(\omega)\right)\right\},(t=1,2, \cdots)$ be a sequence of 4-dimensional random vectors and let $X_{t}, Y_{t}, Z_{t}$ and $W_{t}$ denote the states of players 
$1,2,3$ and 4 at the $t$-th stage respectively, which can be observed by every player. We denote by $X, Y, Z$ and $W$ the state sets of players $1,2,3$ and 4 respectively and suppose that $X, Y, Z$ and $W$ are finite. We use the notations $S=X \times Y \times Z \times W$ and $S_{t}=\left(X_{t}, Y_{t}, Z_{t}, W_{t}\right)$ which are called the state set and the state vector at the $t$-th stage respectively. We denote further by $\xi_{t}$ the sequence of state vectors up to the $t$-th stage, i. e. $\xi_{t}=\left(S_{1}, \cdots, S_{t}\right)$ and denote by $P_{i j k l}^{(t)}\left(\xi_{t}\right)$ the conditional probability of $\xi_{t}$ under the condition that $\theta_{1}=\alpha_{i}, \theta_{2}=\beta_{j}, \theta_{3}=\gamma_{k}$ and $\theta_{4}=\delta_{l}$.

We denote by $J_{i: j}(t)$ the amount of information that player $i$ can obtain by himself about player $j$ 's strategy from the sequence $\xi_{t}$. We define the value as follows:

$$
J_{i: j}(t)=H\left(\theta_{j}\right)-E\left[H\left(\theta_{j} ; \theta_{i}, \xi_{t}\right)\right],
$$

where $H\left(\theta_{j} \mid \theta_{i}, \xi_{t}\right)$ is the conditional entropy of $\theta_{j}$ when $\theta_{i}$ and $\xi_{t}$ are given, i. e.

$$
H\left(\theta_{j} \mid \theta_{i}, \xi_{t}\right)=\sum_{\theta_{j}} P\left(\theta_{j} \mid \theta_{i}, \xi_{t}\right) \log \frac{1}{P\left(\theta_{j} \mid \theta_{i}, \hat{\xi}_{t}\right)}
$$

and $E\left[H\left(\theta_{j} \mid \theta_{i}, \xi_{t}\right)\right]$ means the expectation of $H\left(\theta_{j} \mid \theta_{i}, \hat{\xi}_{t}\right)$, i. e.

$$
E\left[H\left(\theta_{j} \mid \theta_{i}, \hat{\xi}_{t}\right)\right]=\sum_{\theta_{i}} \sum_{\bar{\Sigma}_{t}} P\left(\theta_{i}, \hat{\xi}_{t}\right) H\left(\theta_{j} \mid \theta_{i}, \hat{\xi}_{t}\right) .
$$

Now we suppose that the cooperation between players is the phenomenon such that the players in a group give each other all the information they have. On the other hand, considering the definition (6), we can see that the difference between the two quantities $J_{i: k}(t)$ and $J_{j: k}(t)$ are caused only by the difference between $\theta_{i}$ and $\theta_{j}$. Therefore we define the cooperation as the behaviors that the players in the cooperative group give each other the information about their own strategies. To avoid complexity we study the amount of information that player 1 in cooperation with some others obtains about player 4's strategy from sequential observations. Then we have the following definitions.

Definition 1. $J_{12: 4}(t)$ denotes tha amount of information that player 1 in cooperation with player 2 obtains about player 4's strategy from the sequence $\xi_{t}$ and is defined by

$$
J_{12: 4}(t)=H\left(\theta_{4}\right)-E\left[H\left(\theta_{4} \mid \theta_{1}, \theta_{2}, \xi_{l}\right)\right],
$$

where $H\left(\theta_{4} \mid \theta_{1}, \theta_{2}, \xi_{t}\right)$ means the conditional entropy of $\theta_{4}$ when $\theta_{1}, \theta_{2}$ and $\xi_{t}$ are given. Obviously by (8) we have

$$
J_{21: 4}(t)=J_{12: 4}(t) \text {, }
$$

so we can say that the cooperation is reciprocal. $J_{13: 4}(t)$ is similarly defined as the amount of information that player 1 in cooperation with player 3 obtains on player 4's strategies from $\xi_{t}$.

DEFinition 2. $J_{123: 4}(t)$ denotes the amount of informaiton that player 1 in cooperation with players 2 and 3 obtains about player 4's strategy from $\xi_{t}$ and is defined by

$$
J_{123: 4}(t)=H\left(\theta_{4}\right)-E\left[H\left(\theta_{4} \mid \theta_{1}, \theta_{2}, \theta_{3}, \xi_{t}\right)\right],
$$

where $H\left(\theta_{4} \mid \theta_{1}, \theta_{2}, \theta_{3}, \xi_{t}\right)$ means the conditional entropy of $\theta_{4}$ when $\theta_{1}, \theta_{2}, \theta_{3}$ and $\xi_{t}$ are given. We easily see by (9) that 


$$
J_{123: 4}(t)=J_{2: 1: 4}(t)=J_{312: 4}(t) \text {. }
$$

Concerning the above quantities in Definition 1 and Definition 2 we have the following result.

LEMMA 2. (a) The quantities $J_{1: 4}(t), J_{12: 4}(t), J_{13:}(t)$ and $J_{123: 4}(t)$ are nondecreasing for $t=1,2, \cdots$.

(b) For every $t,(t=1,2, \cdots)$ the following inequalities hold:

$$
\begin{aligned}
H\left(\theta_{4}\right) & \geqq J_{123: 4}(t) \\
& \geqq \max \left\{J_{12: 4}(t), J_{13: 4}(t)\right\} \\
& \geqq \min \left\{J_{12: 4}(t), J_{13: 4}(t)\right\} \\
& \geqq J_{1: 4}(t) .
\end{aligned}
$$

PROOF. The proof of the lemma is clear from the elementary properties of the conditional entropy.

From (b) of the lemma we can say that it is better for player 1 to cooperate with some others. We call the value $H\left(\theta_{4}\right)$ the total information on the parameter $\theta_{4}$.

Now we show some sufficient conditions under which player 1 in cooperation with some others can obtain the total information on $\theta_{4}$.

THEOREM 4. (a) If for every $i, j, j^{\prime}, k, k^{\prime}, l$ and $l^{\prime} \neq l$

$$
\lim _{t \rightarrow \infty} \sum_{\xi_{t}}\left\{P_{i j k l}^{(t)}\left(\xi_{t}\right) P_{i j^{\prime} k^{\prime} l}^{(t)},\left(\xi_{t}\right)\right\}^{1 / 2}=0
$$

then

$$
\lim _{t \rightarrow \infty} J_{1: 4}(t)=H\left(\theta_{4}\right) .
$$

(b) If for every $i, j, k, k^{\prime}, l$ and $l^{\prime} \neq l$

$$
\lim _{t \rightarrow \infty} \sum_{\xi_{t}}\left\{P_{i j k l}^{(t)}\left(\xi_{t}\right) P_{i j k^{\prime} l^{\prime}}^{(t)}\left(\xi_{t}\right)\right\}^{1 / 2}=0,
$$

then

$$
\lim _{t \rightarrow \infty} J_{12: 4}(t)=H\left(\theta_{4}\right) .
$$

(c) If for every $i, j, k, l$ and $l^{\prime} \neq l$

$$
\lim _{t \rightarrow \infty} \sum_{\xi_{t}}\left\{P_{i j k l}^{(t)}\left(\hat{\xi}_{t}\right) P_{i j k l}^{(t)}\left(\xi_{t}\right)\right\}^{1 / 2}=0
$$

then

$$
\lim _{t \rightarrow \infty} J_{123: 4}(t)=H\left(\theta_{4}\right) .
$$

Proof. We prove only (b). (a) and (c) are proved in the same manner. By Definition 1 we have

$$
\begin{aligned}
& E\left[H\left(\theta_{4} \mid \theta_{1}, \theta_{2}, \xi_{t}\right)\right] \\
= & \sum_{\theta_{1}} \sum_{\theta_{2}} \sum_{\xi_{t}} P\left(\theta_{1}, \theta_{2}, \xi_{t}\right) \sum_{\theta_{4}} P\left(\theta_{4} \mid \theta_{1}, \theta_{2}, \xi_{t}\right) \log \frac{1}{P\left(\theta_{4} \mid \theta_{1}, \theta_{2}, \xi_{t}\right)}
\end{aligned}
$$




$$
=\sum_{\theta_{1}} \sum_{\theta_{2}} \sum_{\bar{s}_{t}} \sum_{\theta_{4^{\prime}}^{\prime}} P\left(\theta_{1}, \theta_{2}, \theta_{4}^{\prime}, \xi_{t}\right) \sum_{\theta_{4}} P\left(\theta_{4} \mid \theta_{1}, \theta_{2}, \xi_{t}\right) \log \frac{1}{P\left(\theta_{4} \mid \theta_{1}, \theta_{2}, \xi_{t}\right)}
$$

Then, applying Lemma 1 and the Bayes' theorem, we have the following for a positive constant $C$ :

$$
\begin{aligned}
& E\left[H\left(\theta_{4} \mid \theta_{1}, \theta_{2}, \xi_{t}\right)\right] \\
& \leqq C \sum_{\theta_{1}} \sum_{\theta_{2}} \sum_{\overline{⿳ 亠 口}} \sum_{\theta_{4^{\prime}}} P\left(\theta_{1}, \theta_{2}, \theta_{4}^{\prime}, \xi_{t}\right) \sum_{\theta_{4} \neq \theta_{4^{\prime}}} \sqrt{P\left(\theta_{4} \mid \theta_{1}, \theta_{2}, \overline{\xi_{t}}\right)} \\
& =C \sum_{\theta_{1}} \sum_{\theta_{2}} \sum_{\xi_{i} t} \sum_{\theta_{4^{\prime}}} P\left(\theta_{1}, \theta_{2}, \theta_{4}^{\prime}, \xi_{t}\right) \sum_{\theta_{4}^{\neq} \neq \theta_{t^{\prime}}} \frac{\sqrt{P\left(\theta_{1}, \theta_{2}, \theta_{4}, \xi_{t}\right)}}{\sqrt{P} \bar{P}\left(\overline{\theta_{1}}, \theta_{2}, \xi_{t}\right)} \\
& \leqq C \sum_{\theta_{1}} \sum_{\theta_{2}} \sum_{\xi_{t}} \sum_{\theta_{4^{\prime}}} \sum_{\theta_{4} \neq \theta_{4^{\prime}}} \sqrt{P\left(\theta_{1}, \theta_{2}, \theta_{4}^{\prime}, \xi_{t}\right) \overline{P\left(\theta_{1}, \theta_{2}, \theta_{4}, \xi_{t}\right)}} \\
& \leqq C \sum_{\theta_{1}} \sum_{\theta_{2}} \sum_{\xi_{t}} \sum_{\theta_{4^{\prime}}} \sum_{\theta_{4} \neq \theta_{4^{\prime}}} \sum_{\theta_{3}} \sum_{\theta_{3^{\prime}}} \sqrt{P\left(\theta_{1}, \theta_{2}, \theta_{3}^{\prime}, \theta_{4}^{\prime}, \xi_{t}\right) P\left(\theta_{1}, \theta_{2}, \theta_{3}, \theta_{4}, \xi_{t}\right)} .
\end{aligned}
$$

Under the conditions that $\theta_{1}=\alpha_{i}, \theta_{2}=\beta_{j}, \theta_{3}=\gamma_{k}$ and $\theta_{4}=\delta_{l}$, we have

$$
P\left(\theta_{1}, \theta_{2}, \theta_{3}, \theta_{4}, \xi_{t}\right)=p_{i j k l} P_{i j k l}^{(t)}\left(\xi_{t}\right) \text {. }
$$

Then it holds that

$$
\begin{aligned}
& E\left[H\left(\theta_{4} \mid \theta_{1}, \theta_{2}, \xi_{t}\right)\right] \\
\leqq & C \sum_{i} \sum_{j} \sum_{k} \sum_{k^{\prime}} \sum_{l^{\prime}} \sum_{l^{\prime} \neq l} \sum_{\xi t}\left\{P_{i j k l}^{(t)}\left(\xi_{t}\right) P_{i j k^{\prime} l}^{(t)}\left(\xi_{t}\right)\right\}^{1 / 2} .
\end{aligned}
$$

This proves the conclusion (b).

Clearly the condition in (a) satisfies that in (b), and also the condition in (b) satisfies that in (c). When the state vectors $\left\{S_{t}\right\}$ are independent and identically distributed under conditions that $\theta_{1}=\alpha_{i}, \theta_{2}=\beta_{j}, \theta_{3}=\gamma_{k}$ and $\theta_{4}=\delta_{l}$, we have the following result. We put, for every $t(t=1,2, \cdots)$ and $S_{t} \in S$,

$$
\pi\left(S_{t}\right)_{i j k l}=P\left(S_{t} \mid \theta_{1}=\alpha_{i}, \theta_{2}=\beta_{j}, \theta_{3}=\gamma_{k}, \theta_{4}=\delta_{l}\right) .
$$

COROLlaRY. Let $\left\{S_{t}\right\},(t=1,2, \cdots)$ be independent and identically distributed under conditions that $\theta_{1}=\alpha_{i}, \theta_{2}=\beta_{j}, \theta_{3}=\gamma_{k}$ and $\theta_{4}=\delta_{l}$. Then we have the following results:

(a) If for every $i, j, j^{\prime}, k, k^{\prime}, l$ and $l^{\prime} \neq l$

$$
\sum_{s \in S} \sqrt{\pi(s)_{i j k l} \pi(s)_{i j^{\prime} k^{\prime} l^{\prime}}}<1,
$$

then

$$
\lim _{t \rightarrow \infty} J_{1: 4}(t)=H\left(\theta_{4}\right) .
$$

(b) If for every $i, j, k, k^{\prime}, l$ and $l^{\prime} \neq l$

$$
\sum_{s \in S} \sqrt{\pi(s)_{i j k l} \pi(s)_{i j k^{\prime} l^{\prime}}}<1,
$$

then

$$
\lim _{t \rightarrow \infty} J_{12: 4}(t)=H\left(\theta_{4}\right) .
$$


(c) If for every $i, j, k, l$ and $l^{\prime} \neq l$

$$
\sum_{s=S} \sqrt{\pi(s)_{i j k l} \pi(s)_{i j k l^{\prime}}}<1,
$$

then

$$
\lim _{t \rightarrow \infty} J_{12: 3:}(t)=H\left(\theta_{4}\right) .
$$

Proof. We prove only (b), for (a) and (c) are proved similarly. Since $\left\{S_{t}\right\}$, $(t=1,2, \cdots)$ are independent and identically distributed under conditions $\theta_{1}=\alpha_{i}, \theta_{2}=\beta_{j}$, $\theta_{3}=\gamma_{k}$ and $\theta_{4}=\delta_{l}$, we obtain

$$
P\left(\xi_{t}\right)_{i j k l}^{(t)}=\prod_{i=1}^{t} \pi\left(S_{\nu}\right)_{i j k l}
$$

Thus for every $i, j, k, k^{\prime}, l$ and $l^{\prime} \neq l$

$$
\begin{aligned}
& \sum_{\hat{\xi} t} \sqrt{P_{i j k l}^{(t)}\left(\xi_{t}\right) \overline{P_{i j k^{\prime} l^{\prime}}^{(t)}\left(\xi_{t}\right)}} \\
= & \sum_{S_{1}} \sum_{S_{2}} \cdots \sum_{S_{t}} \prod_{\nu=1}^{t} \sqrt{\pi\left(S_{\nu}\right)_{i j k l} \pi\left(S_{2}\right)_{i j k^{\prime} l^{\prime}}} \\
= & \left(\sum_{s \in S} \sqrt{\pi(s)_{i j k l} \pi(s)_{i j k^{\prime} l^{\prime}}}\right)^{t} .
\end{aligned}
$$

By the assumption this proves the conclusion (b).

Now we consider the optimal cooperations for player 1 when he is not allowed to cooperate with more than one players. Then the problem for player 1 is with which player he should cooperate, player 2 or player 3 . There are four cases to consider.

$$
\begin{array}{ll}
(\mathrm{A}-1) & \lim _{t \rightarrow \infty} J_{12: 4}(t)>\lim _{t \rightarrow \infty} J_{13: 4}(t) . \\
(\mathrm{A}-2) & \lim _{t \rightarrow \infty} J_{12: 4}(t)=\lim _{t \rightarrow \infty} J_{13: 4}(t) \text { and } \\
& J_{12: 4}(t)>J_{13: 4}(t) \quad \text { for all } t=1,2, \cdots . \\
\text { (B-1) } & \lim _{t \rightarrow \infty} J_{12::}(t)<\lim _{t \rightarrow \infty} J_{13: 4}(t) . \\
\text { (B-2) } & \lim _{t \rightarrow \infty} J_{12: 4}(t)=\lim _{t \rightarrow \infty} J_{13: 4}(t) \text { and } \\
& J_{12: 4}(t)<J_{13: 4}(t) \quad \text { for all } t=1,2, \cdots .
\end{array}
$$

We can say that in the case $(\mathrm{A}-1)$ or $(\mathrm{A}-2)$ player 1 should cooperate with player 2 but in the case $(\mathrm{B}-1)$ or $(\mathrm{B}-2)$ he should do with player 3 . We consider this problem in the following simple numerical models. Let $a$ and $b$ be the strategies of all the players and let $P\left(\theta_{i}=a\right)=P\left(\theta_{i}=b\right)=0.5$ for all $i=1,2,3$ and 4 . Therefore $H\left(\theta_{i}\right)$, the total information on $\theta_{i}$, is equal to 1 . Let $\{0,1\}$ be the state set of all the players, then we can denote $S=\{0,1\} \times\{0,1\} \times\{0,1\} \times\{0,1\}$, where $s=\left(s_{1}, s_{2}, s_{3}, s_{4}\right) \in S$ means that the states of players $1,2,3$ and 4 are $s_{1}, s_{2}, s_{3}$ and $s_{4}$ respectively. We suppose that the sequence of state vectors $\left\{S_{t}\right\}$ are independent and identically distributed under each condition that $\theta_{1}=\alpha_{i}, \theta_{2}=\beta_{j}, \theta_{3}=\gamma_{k}$ and $\theta_{4}=\delta_{l}$. We show three numerical 
models whose conditional probabilities $\left\{\pi(s)_{i j k l}\right\}$ of states are given by numerical tables 1-1, 2-1 and 3-1. In those tables each row denotes the conditional probabilities of states when the values of $\theta_{1}, \theta_{2}, \theta_{3}$ and $\theta_{\frac{1}{4}}$ are given. For example, (4,9)-element in Table 1-1 (i.e. the value at 4 -th row and 9 -th column, which is 0.04 ) means the conditional probability of the state $(0,0,0,1)$ when $\theta_{1}=b, \theta_{2}=b, \theta_{3}=a$ and $\theta_{4}=a$. According to each Table $N-1(N=1,2,3)$ we calculated the quantities $J_{12: t}(t)$ and $J_{13: 4}(t)$. The values up to the 3 rd stage are shown in Table $N-2$. Then we can say that up to the 3rd stage player1 should cooperate with player 2 in the 1 st model given by Table 1-1, but he should cooperate with player 3 in the 2 nd and 3 rd models given by Table 2-1 and Table 3-1 respectively. Furthermore we can see easily that for every $i, j, k, k^{\prime}, l$ and $l^{\prime} \neq l, \sum_{s \in S} \sqrt{\pi(s)_{i j k l} \pi(s)_{i j k^{\prime} l^{\prime}}}<1$ and for every $i, j, j^{\prime}, k, l$ and $l^{\prime} \neq l \sum_{s \in S} \sqrt{\pi(s)_{i j k l} \pi(s)_{i j^{\prime} k l^{\prime}}}$ $<1$. Thus by Corollary we obtain

$$
\lim _{t \rightarrow \infty} J_{12: 4}(t)=\lim _{t \rightarrow \infty} J_{13: 4}(t)=H\left(\theta_{4}\right) .
$$

Considering these results, we can say that the first model is an example of the case $(\mathrm{A}-2)$ and the last two models are examples of the case (B-2).

Table 1-1

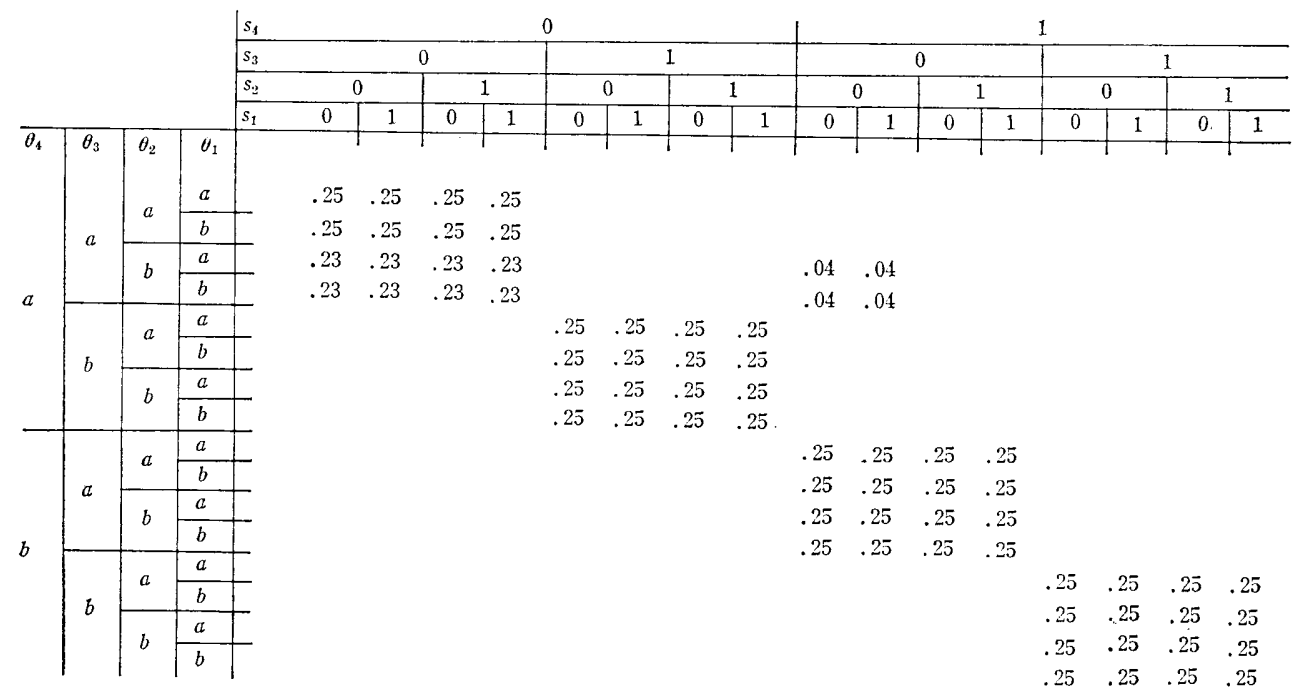

Table 1-2

\begin{tabular}{c|c|c|c} 
& $t=1$ & $t=2$ & $t=3$ \\
\hline$I_{12: 4}(t)$ & 0.95804 & 0.99460 & 0.99940 \\
\hline$I_{13: 4}(t)$ & 0.94857 & 0.99391 & 0.99934 \\
\hline$I_{12: 4}(t)-I_{15: 4}(t)$ & 0.00947 & 0.00079 & 0.00006
\end{tabular}


Table 2-1

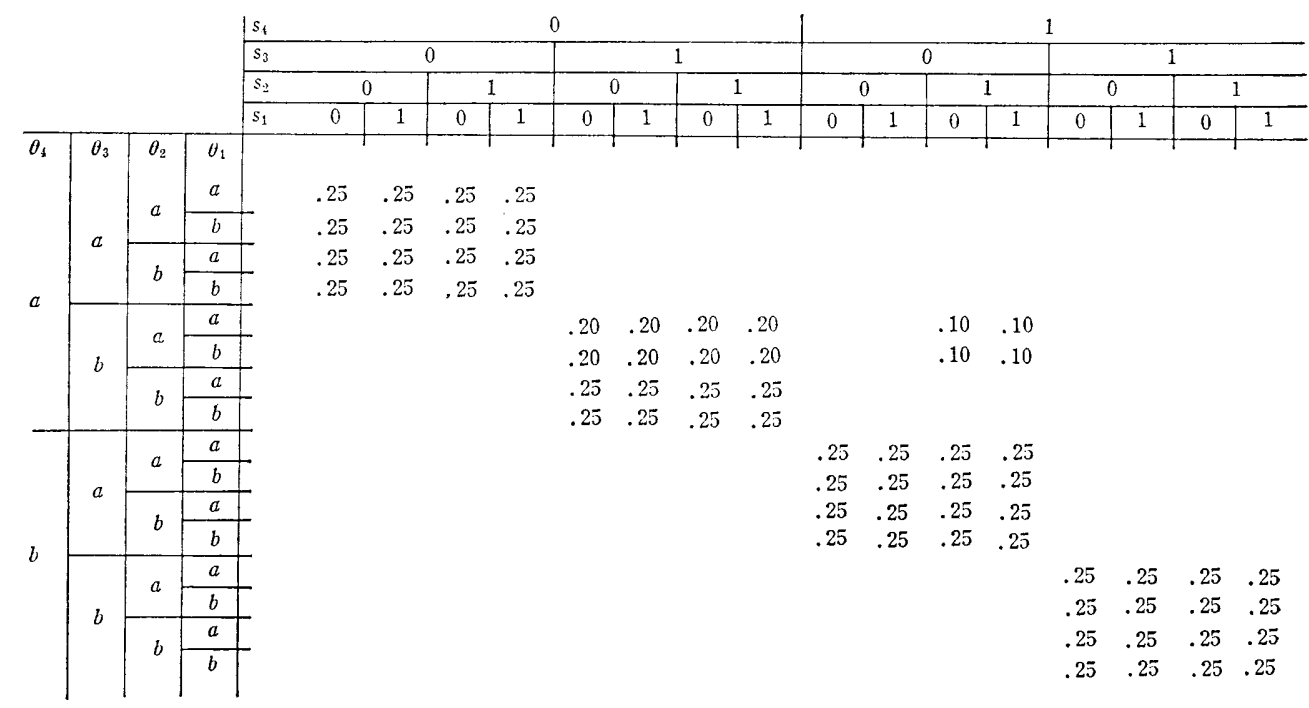

Table 2-2

\begin{tabular}{c|c|c|c} 
& \multicolumn{1}{|c|}{$t=1$} & \multicolumn{1}{c|}{$t=2$} & \multicolumn{1}{c}{$t=3$} \\
\hline$I_{12: 4}(t)$ & 0.92448 & 0.97902 & 0.99455 \\
\hline$I_{13: 4}(t)$ & 1.00000 & 1.00000 & 1.00000 \\
\hline$I_{12: 4}(t)-I_{13: 4}(t)$ & -0.07552 & -0.02098 & -0.00545
\end{tabular}

\section{Discussion.}

In playing a game it is essential for every player to estimate subsequent behaviors of the other players by the information on their parameters obtained from observations on their past behaviors. A win and reward can be considered only as the results of the games. In the present paper we have studied the games from the viewpoint above, and we consider the hierarchy of games as follows:

(1) One-stage games such as toss-up.

Since there is no observation, the information can not be considered.

(2) Repitition of one-stage games.

Sequential game states are independent and identically distributed. The information on strategies can be obtained and is used to estimate the other players' strategies.

(3) Multistage games such as chess or go.

The present state is dependent on the past states, then the observations contain much more information than that in (b) and it becomes easier for a player to estimate the other players' strategies.

(4) Repitition of multistage games like as trainning of chess or go. 
Table $3-1$

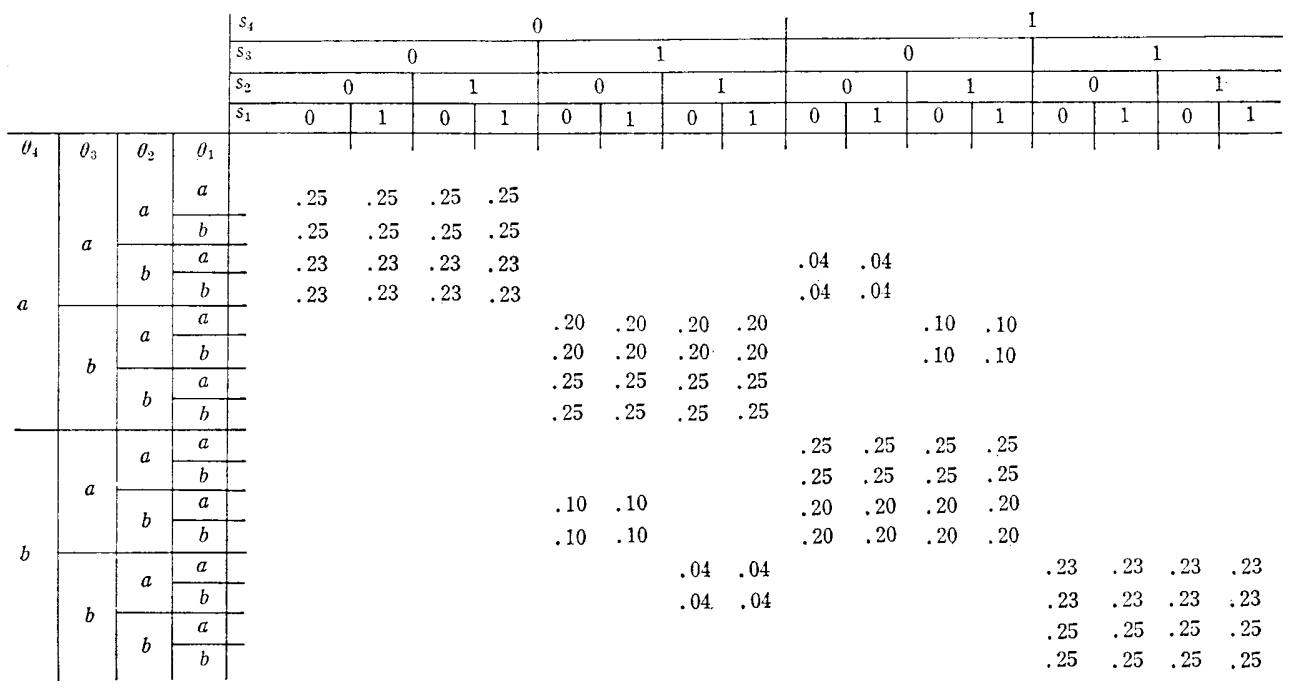

Table $3-2$

\begin{tabular}{c|c|c|c} 
& $t=1$ & $t=2$ & \multicolumn{1}{c}{$t=3$} \\
\hline$I_{12: 4}(t)$ & 0.77095 & 0.94825 & 0.98802 \\
\hline$I_{12: 4}(t)$ & 0.90006 & 0.98807 & 0.99872 \\
\hline$I_{12: 4}(t)-I_{13: 4}(t)$ & -0.12911 & -0.03982 & -0.01070
\end{tabular}

(5) Struggle and revolution in ecological systems.

Every living thing obtain information on environment and learn adaptation to it. On the other hand they sometimes compete and sometimes cooperate each other. Thus these systems can be considered as long-term games.

We have studied the games of (3) in non-cooperative and cooperative cases. We also studied the games of(2) as the special cases of (3) such that the state vectors were independent.

Furthermore we consider that the theory of information games will be applicable to the brain information processing and the learning precesses in the following senses:

(a) To recognize the object, the human sense of vision, auditory, touch, taste and smell are processed and cooperated in the brain. Unified information processing of the five senses in human brain is considered as a model of cooperative game systems.

(b) The game system is similar to learning system in the process of obtaining information. The differece between the both systems is only that a game player makes an effort to keep his parameter secret from his opponents, but a leaner is reinforced by his teacher to obtain information much more and expects that his learning states become known to the teacher. 


\section{References}

[1] A. RÉYYI, "On the amount of information concerning an unknown parameter in a sequence of observations", Publ. of Math. Inst. of Hungarian Acad. Sci., 9 (1964) 617-624.

[2] A. RÉNYI, "On some basic problems of statistics from the point of view of information theory", Proceedings of 5-th Berkeley Symposium, Vol. 1 (1967) 531-543.

[3- J.F. Korsh, "On decisions and information concerning an unknown parameter", Infor. mation and Control, 16 (1970) 123-127.

[4 S. KA.vō and Y. KAI, "Information theoretical approaches in game theory", Bull. of Math. Stat., 19 (1979) 37-43.

[5] S. GuAsu, "Information theory with applications", McGraw-Hill, (1977). 